\title{
FORMATION AND IDENTIFICATION OF NOVEL DERIVATIVES OF PRIMARY AMINE AND ZWITTERIONIC DRUGS
}

Jordan Ash, Logan Hickey and John Goodpaster*

Forensic and Investigative Sciences Program

Indiana University - Purdue University Indianapolis (IUPUI)

402 North Blackford Street, LD 326

Indianapolis, IN 46202

KEY WORDS: forensic science, drugs of abuse, controlled substances, gas chromatography, mass spectrometry, derivatives, derivatization, gabapentin, lorazepam, vigabatrin, pregabalin, clorazepate, amphetamine, $2 \mathrm{C}-\mathrm{I}$

This is the author's manuscript of the article published in final edited form as:

Ash, J., Hickey, L., \& Goodpaster, J. (2018). Formation and identification of novel derivatives of primary amine and zwitterionic drugs. Forensic Chemistry. https://doi.org/10.1016/j.forc.2018.07.004 


\begin{abstract}
Gas chromatography-mass spectrometry (GC-MS) is a "workhorse" in the analysis of controlled substances in forensic laboratories. However, many drugs are not amenable to GC-MS due to thermal instability, non-ideal interactions in the column, or both. To improve the suitability of a molecule for analysis by GC-MS, derivatization can be employed. Derivatization replaces a labile hydrogen in the analyte molecule with a more stable functional group. In this paper, three different derivatization agents were tested for effectiveness with two classes of drugs: primary amines (i.e., amphetamine and 2C-I) and zwitterions (i.e., gabapentin, lorazepam, vigabatrin, pregabalin, and clorazepate). Trifluoroacetic anhydride (TFAA) was used as an acylating agent and N,O-bis(trimethylsilyl)trifluoroacetamide (BSTFA) was used as a silylating agent. Dimethylformamide-dimethyl acetal (DMF-DMA), which has not been previously used for derivatization of drugs, was used as an alkylating agent. DMF-DMA was found to form dimethylaminomethylene derivatives with several primary amines and zwitterions. Amphetamine, $2 \mathrm{C}-\mathrm{I}$, gabapentin, and lorazepam were all detected in their underivatized form but generally suffered from peak asymmetry and band broadening. Derivatization resulted in drastic improvements in their chromatographic behavior. Vigabatrin, clorazepate and pregabalin were not detectable in their underivatized form. However, the trimethylsilyl (TMS) derivative of clorazepate was readily detected by GC-MS, as were the TMS and trifluoroacetyl (TFA) derivatives of vigabatrin. Derivatization of pregabalin was not successful, resulting in multiple chromatographic peaks with each derivatization agent. The mass spectra of several derivatives were not found in commercially available mass spectral databases. Hence, those spectra are reported here with interpretation of their fragmentation.
\end{abstract}




\section{Introduction}

A wide variety of controlled substances are submitted to forensic laboratories, making up a significant portion of all samples analyzed. However, some of these controlled substances are difficult to analyze by the typical means employed in forensic science laboratories. For example, gas chromatography-mass spectrometry (GC-MS) is a "workhorse" technique for drug chemistry units. However, not all drugs are amenable to GC-MS. For a substance to be analyzed by GC-MS, it must be volatile (i.e., exhibit a vapor pressure of at least one torr in the heated GC inlet) and thermally stable (to at least 200 degrees Celsius). Additionally, some analytes exhibit poor chromatography due to non-ideal interactions with the stationary phase of the column. For example, zwitterions (which have at least one positively and one negatively charged functional group) and basic molecules such as amines are notorious for poor performance in GC.

This paper will discuss the analysis and derivatization of primary amines as well as two benzodiazepines and several other zwitterionic drugs, the structures of which are shown in Fig. 1, below.

(n)




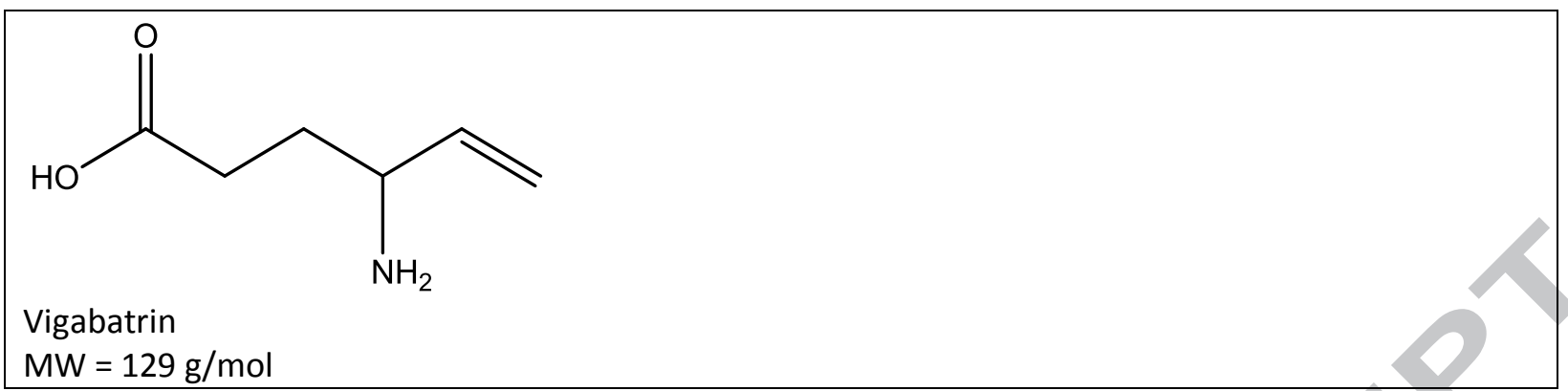

Figure 1. Chemical structures of the drugs analyzed in this work.

A common way to increase the volatility, thermal stability, and chromatographic performance of an analyte is via derivatization [1, 2]. The process of derivatization replaces a labile hydrogen on the analyte molecule with a new functional group that increases the stability of the molecule. Derivatives are most commonly formed via acylation, silyation and alkylation reactions. Acylation reagents include anhydrides of carboxylic acids (e.g., trifluoroacetic acid anhydride or TFAA) and acyl halides (e.g., alkylchloroformates). Silyation reagents include silyl chlorides (e.g., trimethylsilylchloride or TMCS) or silyl acetamides (N,O-Bis(trimethylsilyl)trifluoroacetamide or BSTFA). Alkylation reagents include transesterification reagents (e.g., $\mathrm{KOH}$ and methanol), carbonyl compounds that produce imines/enamines from primary and secondary amines, and formation of Schiff bases using reagents like DMF-DMA.

General forms of these reactions are shown in Figure 2.

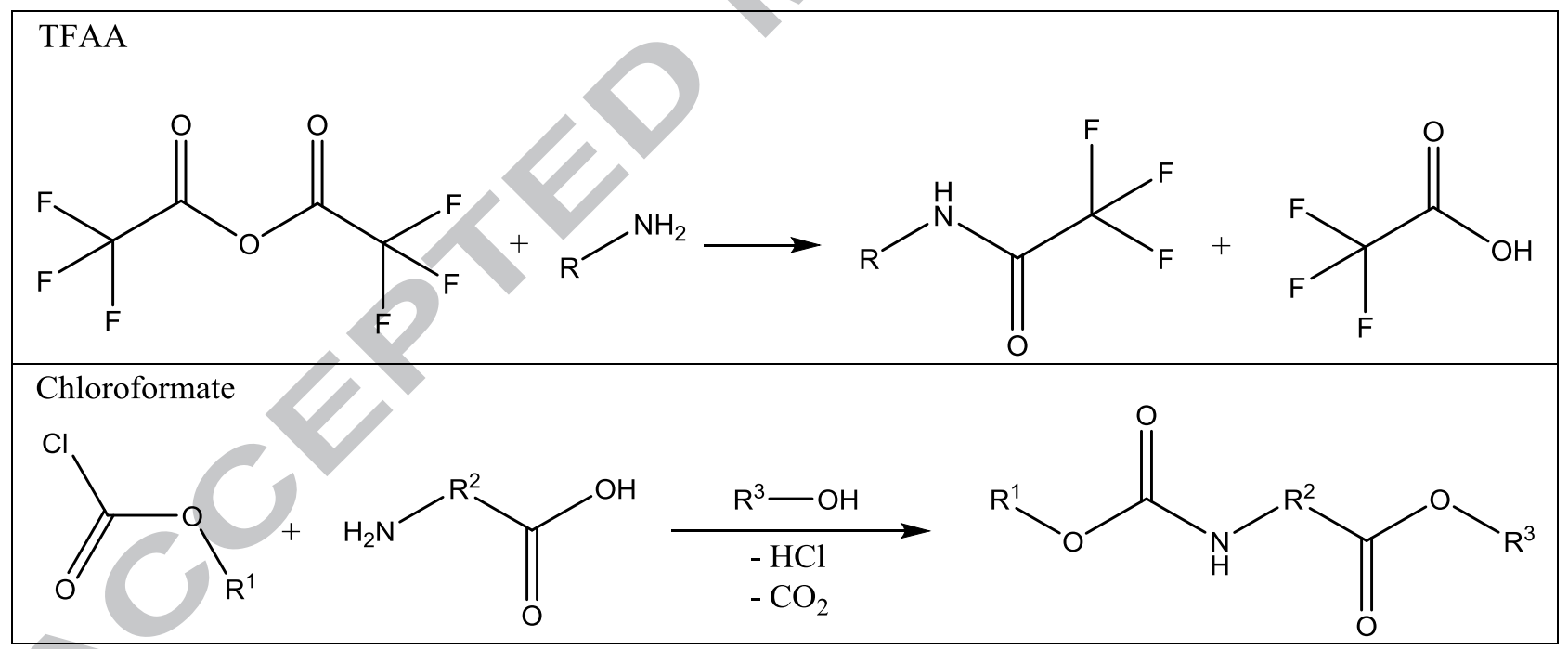




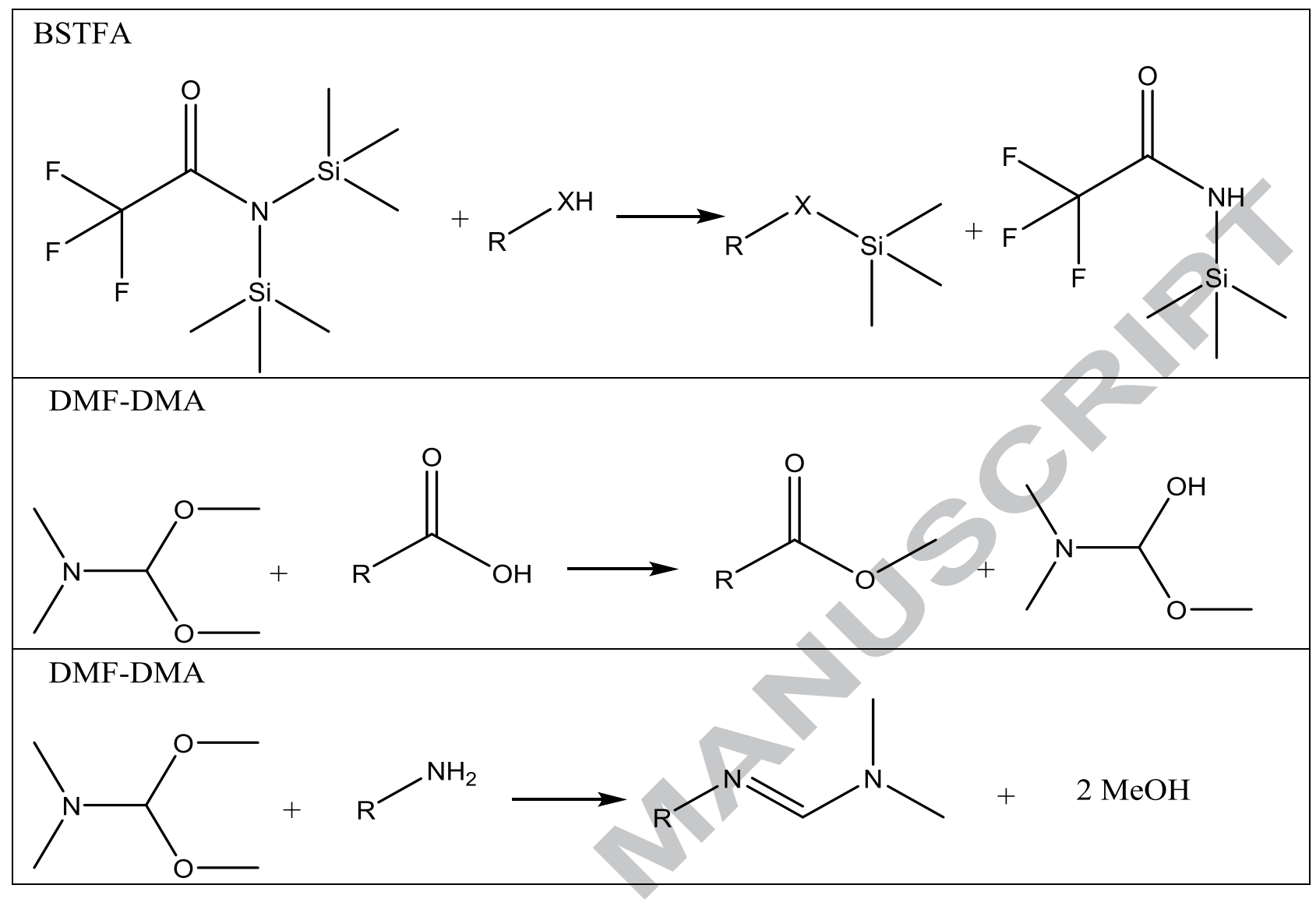

Figure 2. Generalized reactions between TFAA and a primary amine, an alkylchloroformate and a zwitterion, BSTFA where $\mathrm{X}$ is a nitrogen or an oxygen atom, DMF-DMA and a carboxylic acid, and DMFDMA and a primary amine.

As shown in Figure 2, amine and hydroxylamine compounds can be derivatized via acylation, which is the replacement of a labile hydrogen with an acyl group [ $\underline{3}, \underline{4}$ ]. Trifluoroacetic anhydride (TFAA) is a common acylation agent [ $\underline{3}, \underline{5}]$. In this reaction, the amine hydrogen is removed and replaced with a trifluoroacyl group. Chloroformates can be used in acylation reactions with zwitterions. Although these reagents will not be discussed in this paper, ethyl chloroformate derivatives have been formed from pregabalin as a solid, in urine, and in pharmaceuticals [ $[\underline{6}, \underline{7}]$. Hexyl chloroformate derivatives have been formed from gabapentin, vigabatrin, and pregabalin in serum [8].

Carboxylic and phosphonic acids can be derivatized by silylation, which is the replacement of a labile hydrogen with a trimethylsilyl (TMS) group [4, ㅇ]. N,O-bis(trimethylsilyl)trifluoroacetamide (BSTFA) with trimethylchlorosilane (TMCS) is a commonly used silylating agent. Other silylation agents are available which attach larger silyl groups. For example, methyl-N-t-butyldimethylsilyltrifluoroacetamide (MTBSTFA) replaces labile hydrogens with t-butyldimethylsilyl groups. While MTBSTFA derivatives require more time to form, they are more stable [10].

Alkylation is the replacement of a labile hydrogen with an alkyl group []]. Alkylation can be achieved through base- or acid-catalyzed transesterification using reagents such as $\mathrm{KOH} / \mathrm{methanol}$ or $\mathrm{H}_{2} \mathrm{SO}_{4}$ /methanol followed by a liquid-liquid extraction. In contrast, $\mathrm{N}, \mathrm{N}$-dimethylformamide dimethyl acetal (DMF-DMA) is an alkylation reagent that does not require catalysis and is compatible with most 
organic solvents. DMF-DMA has been used for the alkylation of fatty acids [11], as well as the derivatization of amino acids $[\underline{12}, \underline{13}]$ and heterocyclic amines [14]. Primary amines underwent Schiff base condensation, meaning that they lost two hydrogens, which were replaced with a dimethylaminomethylene (DMAM) group. A carbon-nitrogen double bond was thus formed, resulting in an imine [12-14]. In the case of amino acids, the carboxyl moiety is methylated as well, to form a methyl ester $[\underline{12}, \underline{13}]$. Though well known for those applications, the use of DMF-DMA for the derivatization of drugs of abuse has not been previously reported.

Given the difficulty of analyzing these drugs via GC-MS, several spectrophotometric, spectrofluorometric, and liquid chromatographic methods have been reported for the identification of gabapentin, vigabatrin, and pregabalin [15]. Successful analysis of various drugs by GC-MS without derivatization has been reported for $2 \mathrm{C}-\mathrm{I}$ in rat urine [16], gabapentin in human serum [17], and amphetamine [18] and lorazepam [19] in human urine. Amphetamine has been analyzed in hair [20] and urine $[\underline{3}, \underline{21}]$ using TFAA derivatization. $\mathrm{N}$ - N-methyl-bis(trifluoroacetamide) (MBTFA) has been used for the analysis of pure amphetamine samples [ $\underline{5}$ ]. 2C-I has been analyzed in rat urine using TFAA derivatization [16]. Gabapentin has been analyzed following derivatization with N-tertbutyldimethylsilyl-N-methyltrifluoroacetamide (MTBSTFA) in blood plasma and serum [22]. Lorazepam has been analyzed in urine utilizing both MTBSTFA derivatization [23] and a dual derivatization procedure with MTBSTFA and TFAA [24]. The di-TMS derivative of lorazepam has also been formed [25].

Despite these previous efforts, several reactions between drugs and potential derivatization agents have not been explored. For example, the use of DMF-DMA with these drugs has not been previously reported. A summary of previous work in this area appears in Table 1.

[INSERT TABLE 1 HERE]

\section{Experimental}

\subsection{Materials}

Gabapentin, vigabatrin, and $2 \mathrm{C}-\mathrm{I} \mathrm{HCl}$ were purchased from Cayman Chemical (Ann Arbor, Michigan). Lorazepam, pregabalin, amphetamine, dimethylformamide dimethyl acetal (DMF-DMA) and trifluoroacetic anhydride (TFAA) were purchased from Sigma-Aldrich (St. Louis, Missouri). Clorazepate dipotassium was purchased from Grace Chemical (Columbia, Maryland). HPLC grade methanol, Optima acetonitrile, and $\mathrm{N}, \mathrm{O}$-bis(trimethylsilyl)trifluoroacetamide with $1 \%$ trimethylchlorosilane (BSTFA + $1 \%$ TMCS) were purchased from Thermo Fisher Scientific (Waltham, Massachusetts).

\subsection{Sample Preparation}

The drug standards were dissolved in methanol at a concentration of $0.5 \mathrm{mg} / \mathrm{mL}$. When samples were analyzed without derivatization, the solution in methanol was directly injected into the gas chromatograph. Whenever derivatization was employed, additional sample preparation steps had to be taken. A volume of one milliliter of the methanolic drug solution was transferred to an autosampler vial. The methanol was then evaporated using a blow-down apparatus. Two hundred microliters of derivatization agent were then added to the vial and allowed to react at $60^{\circ} \mathrm{C}$ until the solid had completely dissolved. Eight hundred microliters of acetonitrile were then added to bring the total volume back up to one milliliter before injection into the gas chromatograph. 


\subsection{GC-MS Parameters}

An Agilent 6890N gas chromatograph coupled to an Agilent 5975 inert Mass Selective Detector with an attached Gerstel PAL RTC Multi-Purpose Sampler (MPS) was used for all experiments. The column was an Agilent Technologies DB-5MS capillary column with a length of $30 \mathrm{~m}$, a $0.250 \mathrm{~mm}$ inner diameter, and a $0.25 \mu \mathrm{m}$ film thickness. An injection volume of one microliter was used.

The inlet temperature was set to $250^{\circ} \mathrm{C}$ and operated in split mode with a $15: 1$ ratio for amphetamine and in splitless mode for all other analytes. The initial oven temperature of $90^{\circ} \mathrm{C}$ was held for one minute, then the temperature was ramped at $15^{\circ} \mathrm{C} / \mathrm{min}$ to $280^{\circ} \mathrm{C}$ where it was held for one minute. $\mathrm{A}$ speed optimized flow of $2.5 \mathrm{~mL} / \mathrm{min}$ hydrogen was maintained. The mass transfer line was held at $280^{\circ} \mathrm{C}$. The source was kept at $230^{\circ} \mathrm{C}$ and the quadrupoles were kept at $150^{\circ} \mathrm{C}$. A scan range of $\mathrm{m} / \mathrm{z} 40-$ $\mathrm{m} / \mathrm{z} 550$ was used.

Both the NIST/EPA and SWGDRUG mass spectral libraries were used to search all compounds. The fragmentation pattern of all compounds was also determined relative to their chemical structure to confirm their identity. Incomplete derivatizations were identified by peaks corresponding to the retention time of the underivatized drug as well as its mass spectrum.

\subsection{Assessing chromatographic performance.}

The effect of derivatization on sensitivity was monitored by comparing the peak area of an underivatized compound to that of the derivatized compound where both were at the same molar concentration. Peak shape was monitored using the number of theoretical plates $(N)$ and peak asymmetry $\left(A_{s}\right)$. Theoretical plates are given using the following formula:

$N=5.54\left(\frac{t_{r}}{W_{1 / 2}}\right)^{2}$

where $t_{r}$ is the retention time and $W_{1 / 2}$ is the width of the peak at half height. Peak asymmetry was calculated using the following formula:

$A_{s}=\left(\frac{t_{r}-a}{b-t_{r}}\right)$

where $t_{r}$ is the retention time, $a$ is the time of the leading edge of the peak at $10 \%$ height, and $b$ is the time of the falling edge of the peak at $10 \%$ height. Multi-variate analysis was conducted using XLSTAT (Addinsoft, New York, NY), which is an add-in for Microsoft Excel.

\section{Results}

\subsection{Amines: amphetamine and $2 \mathrm{C}-\mathrm{I}$}

As expected, amphetamine could be identified without derivatization. It produced a single chromatographic peak with a mass spectrum that could be identified with a mass spectral library search (Figure 3). 
Derivatization of amphetamine with TFAA produced a much larger, narrower, and more symmetric chromatographic peak compared to a solution of underivatized amphetamine at the same concentration (see Figure 3). Although it has been previously reported [5], the TFA derivative of amphetamine was not in the mass spectra libraries. Hence, the mass spectrum was interpreted by the fragmentation pattern (see Figure 4). The $m / z 118$ fragment resulted from McLafferty rearrangement with charge migration [26]. Alpha cleavage resulted in $\mathrm{m} / \mathrm{z} 91$ (tropylium) and a $\mathrm{m} / \mathrm{z} 140$ peak for the amine side chain.

The reaction of amphetamine with BSTFA was not complete. Although the target compound (amphetamine-TMS) was formed and identified, the underivatized form of the drug was still present in the sample. Extending the reaction time to 60 minutes did not affect the yield of the derivative. Ultimately, BSTFA was deemed unsatisfactory as the derivative must be present in a single chromatographic peak for accurate identification and quantitation (if necessary).

Lastly, the reaction of amphetamine with DMF-DMA resulted in a product that has not been previously reported (Figure 3). Amphetamine followed the same derivatization mechanism as heterocyclic amines with DMF-DMA (see Figure 4) [14]. There was no molecular ion in the mass spectrum, but alpha cleavage at the nitrogen originally present in amphetamine resulted in a fragment at $m / z$ 91 (tropylium) and $m / z$ 99, the base peak. The peak at $m / z 44$ was formed from alpha cleavage from that same nitrogen but cleaved after the carbon in the DMAM.
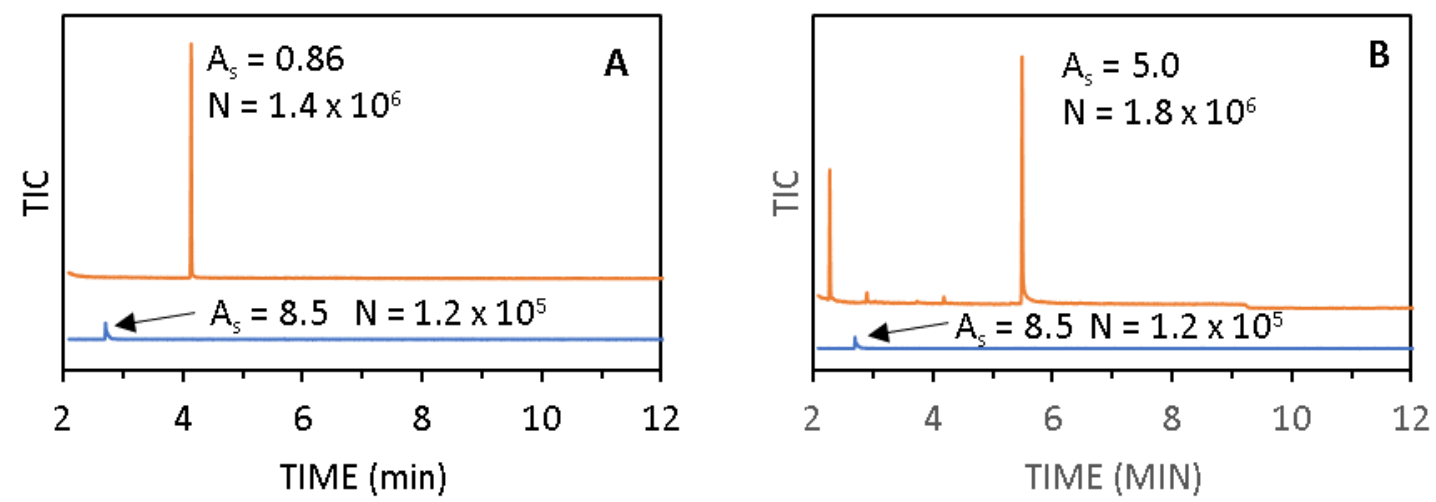

Figure 3. A) TIC of amphetamine TFA (top) and amphetamine underivatized (bottom), B) TIC of amphetamine DMAM (top) and amphetamine underivatized (bottom). $\mathrm{A}_{\mathrm{s}}=$ asymmetry factor. $\mathrm{N}=$ number of theoretical plates.

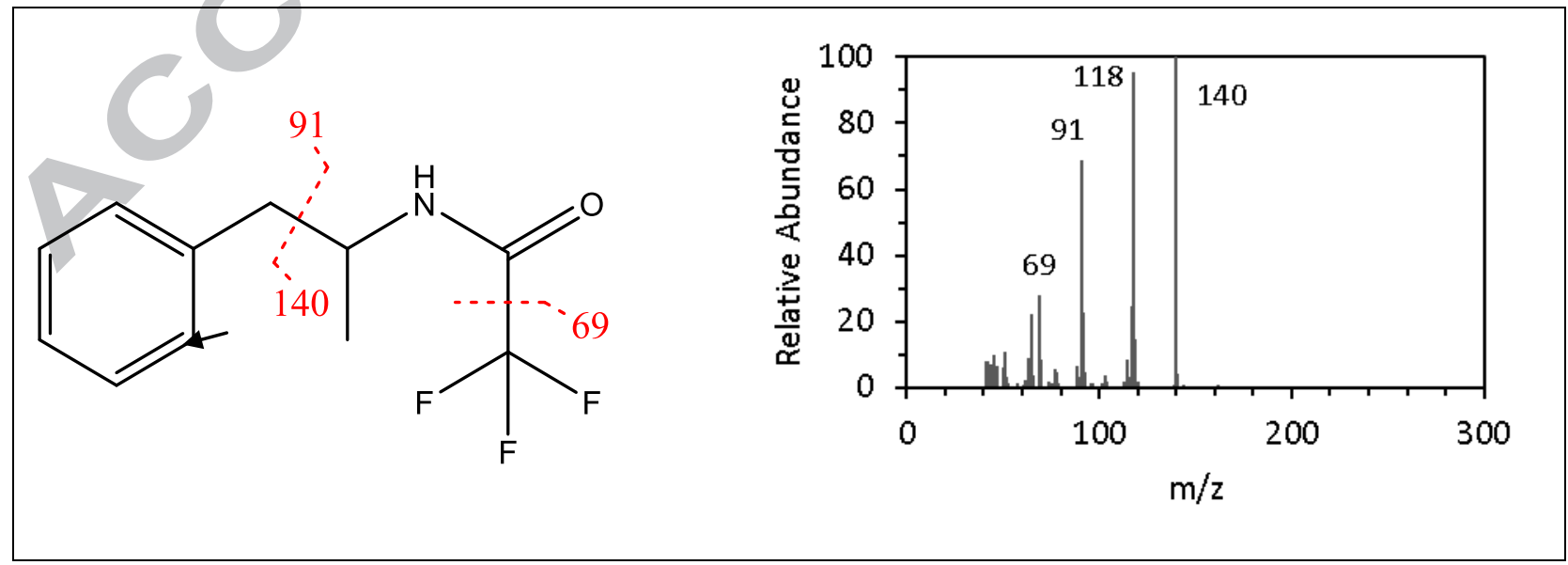



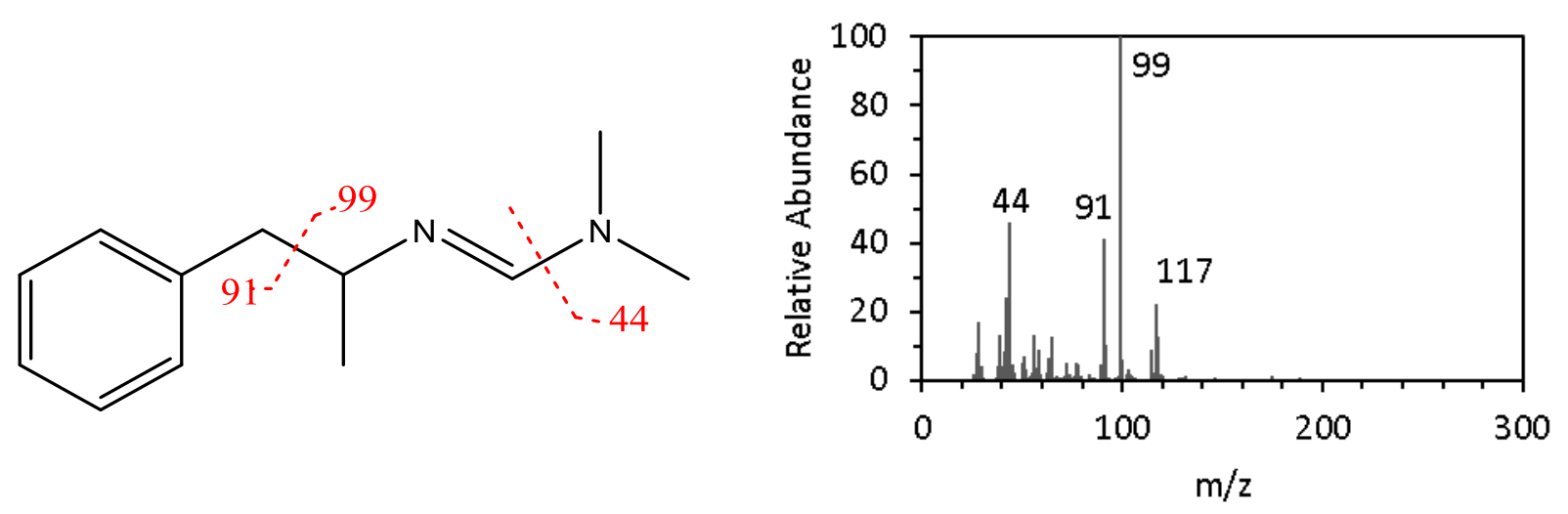

Figure 4. Structure and mass spectrum for amphetamine TFA (MW = 231) and amphetamine DMAM $(\mathrm{MW}=190)$.

Like amphetamine, 2C-I was detected without derivatization, producing a single chromatographic peak with a mass spectrum that was identified with a mass spectral library search (see Figure 5).

The reaction of TFAA with $2 \mathrm{C}-\mathrm{I}$ also resulted in a taller and narrower peak than that produced by underivatized 2C-I (Figure 5). The 2C-I TFA derivative was not in the libraries, but the structure was elucidated by its fragmentation pattern (see Figure 6). After derivatization with TFAA, 2C-I had a molecular ion at $m / z$ 403. The ion at $m / z 290$ was the result of McLafferty rearrangement with charge migration [26]. The molecular ion underwent alpha cleavage at the nitrogen, which produced a fragment at $m / z 277$ and a fragment at $m / z 126$. The fragment at $m / z 247$ was a result of cleavage of two methyl groups from the $m / z 277$ fragment.

Derivatization with BSTFA yielded both a 2C-I derivative with one TMS group on the primary nitrogen and a derivative with two TMS groups on the primary nitrogen. Due to the formation of two derivatives from one analyte, this reaction was determined to be unsatisfactory.

Derivatization of 2C-I with DMF-DMA was, as with amphetamine, a new approach that produced a novel product (see Figure 5). Just like amphetamine, the labile hydrogens on the primary amine in 2C-I were replaced with a DMAM group (see Figure 6). The same alpha cleavage paths resulted in the base peak of $\mathrm{m} / \mathrm{z} 85$ and the characteristic $\mathrm{m} / \mathrm{z}$ 44. There was no molecular ion seen in $2 \mathrm{C}-\mathrm{I}$, however there was a small ion at $m / z 331$. This fragment was a result of the molecular ion losing 31 mass units (methoxy) from the aromatic ring. 

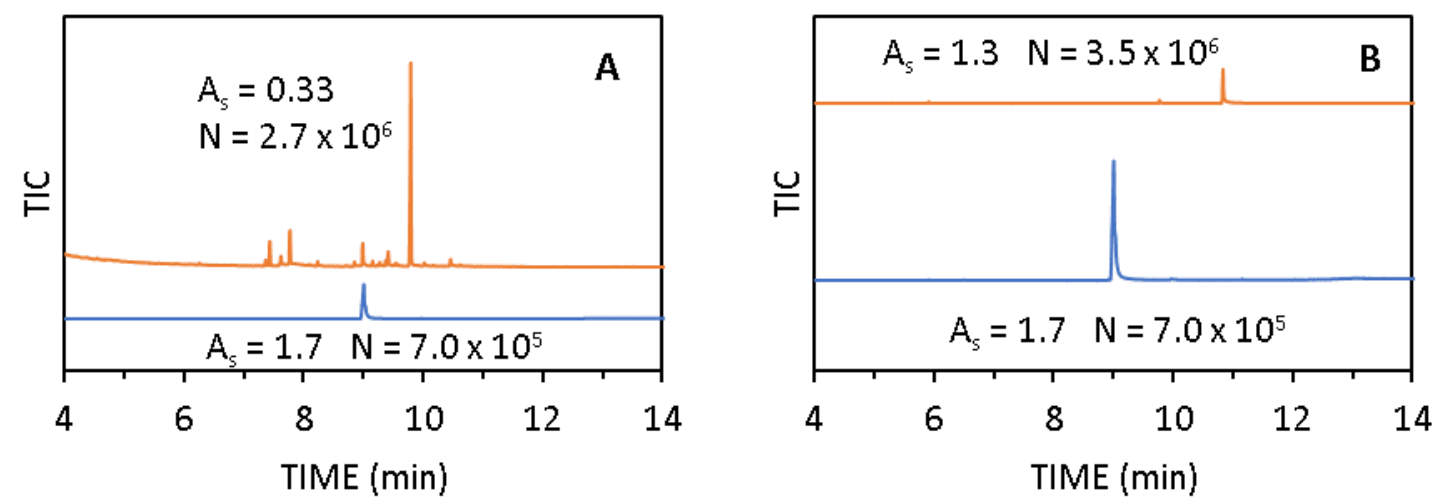

Figure 5. A) TIC of 2C-I TFA (top) and 2C-I underivatized (bottom), B) TIC of 2C-I DMAM (top) and 2C-I underivatized (bottom). $A_{s}=$ asymmetry factor. $\mathrm{N}=$ number of theoretical plates.

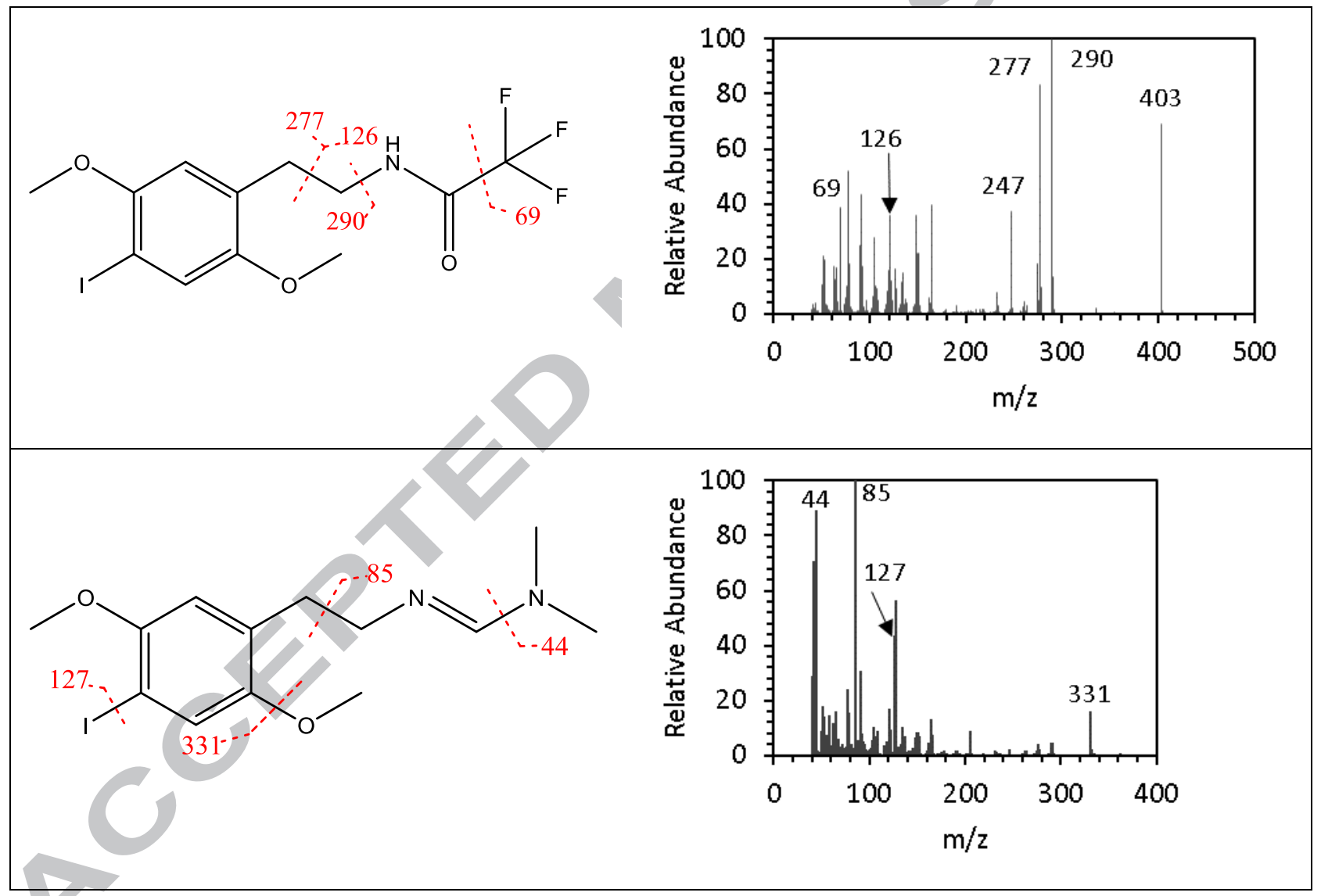

Figure 6. Structure and mass spectrum for 2C-I TFA ( $M W=403)$ and $2 \mathrm{C}-\mathrm{I}$ DMAM $(\mathrm{MW}=362)$.

\subsection{Benzodiazepines: Lorazepam and Clorazepate}

Despite some concerns about the analysis of lorazepam by GC-MS, it produced a single chromatographic peak that was identified with a library search. Derivatization with TFAA was unsuccessful as no derivative was detected. Derivatization with BSTFA produced a single chromatographic peak with a mass spectrum that was identified as the di-TMS derivative of lorazepam by a library search (see Figure 7). 
DMF-DMA derivatization was unsuccessful with lorazepam due to the formation of multiple peaks which could not be attributed to the target derivative.

Clorazepate was not detected in underivatized form. Analysis was also unsuccessful using TFAA derivatization due to the formation of multiple peaks which did not include the target derivative. BSTFA derivatization, however, produced a single chromatographic peak which was identified as the clorazepate-TMS derivative (Figure 7).

The chlorazepate derivative was not in the libraries, but its structure was elucidated by its fragmentation pattern (see Figure 8). The amine group of clorazepate was preferentially silylated. Decarboxylation resulted in the $m / z 45$ and $m / z 341$ ions. The latter ion exhibited an isotopic pattern confirming that one $\mathrm{Cl}$ atom was present. Cleavage of the TMS group resulted in the $\mathrm{m} / z 73$ ion. Derivatization with DMFDMA was unsuccessful for clorazepate as multiple peaks were formed, none of which was attributed to the expected derivative.
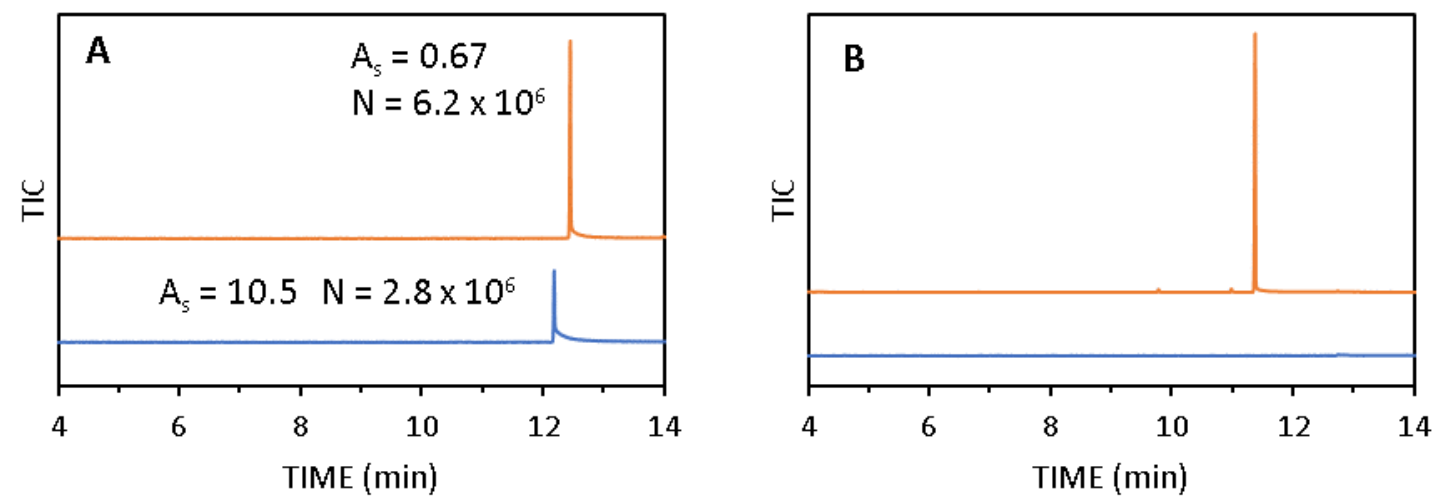

Figure 7. A) TIC of lorazepam TMS (top) and lorazepam underivatized (bottom), B) TIC of clorazepate TMS (top) and clorazepate underivatized (bottom). $A_{s}=$ asymmetry factor. $N=$ number of theoretical plates.

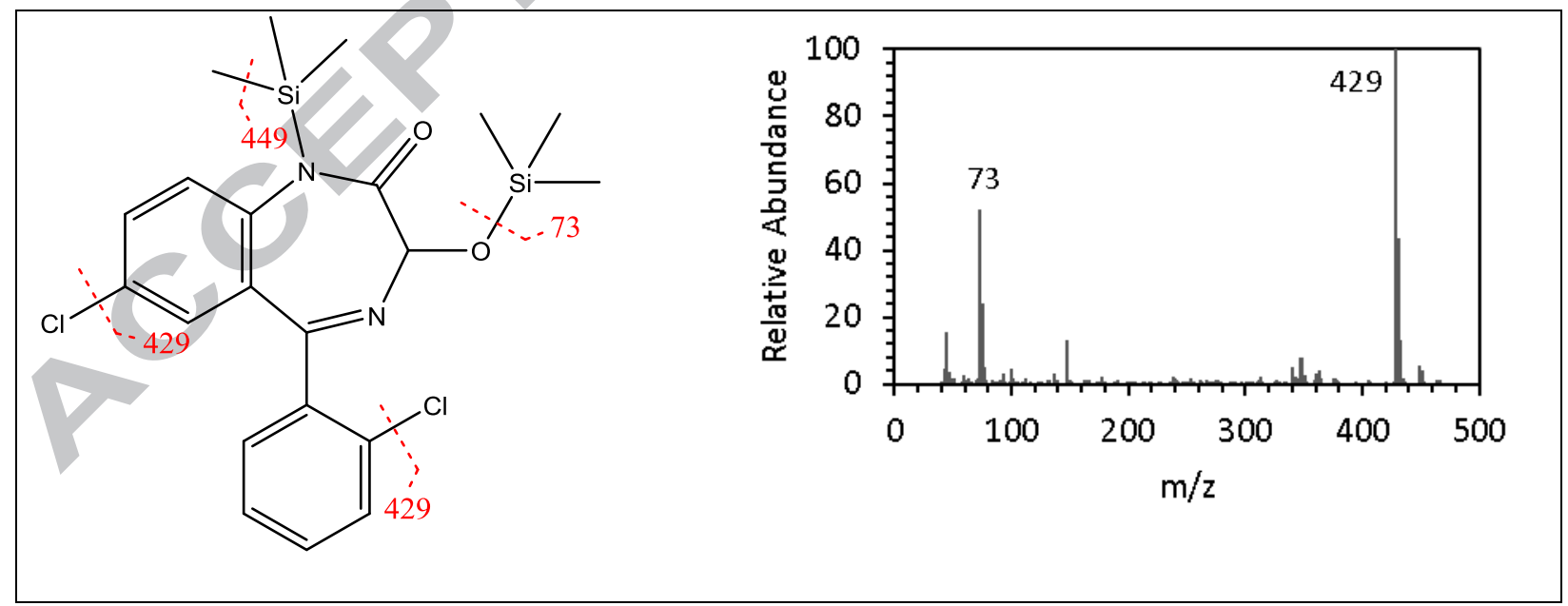




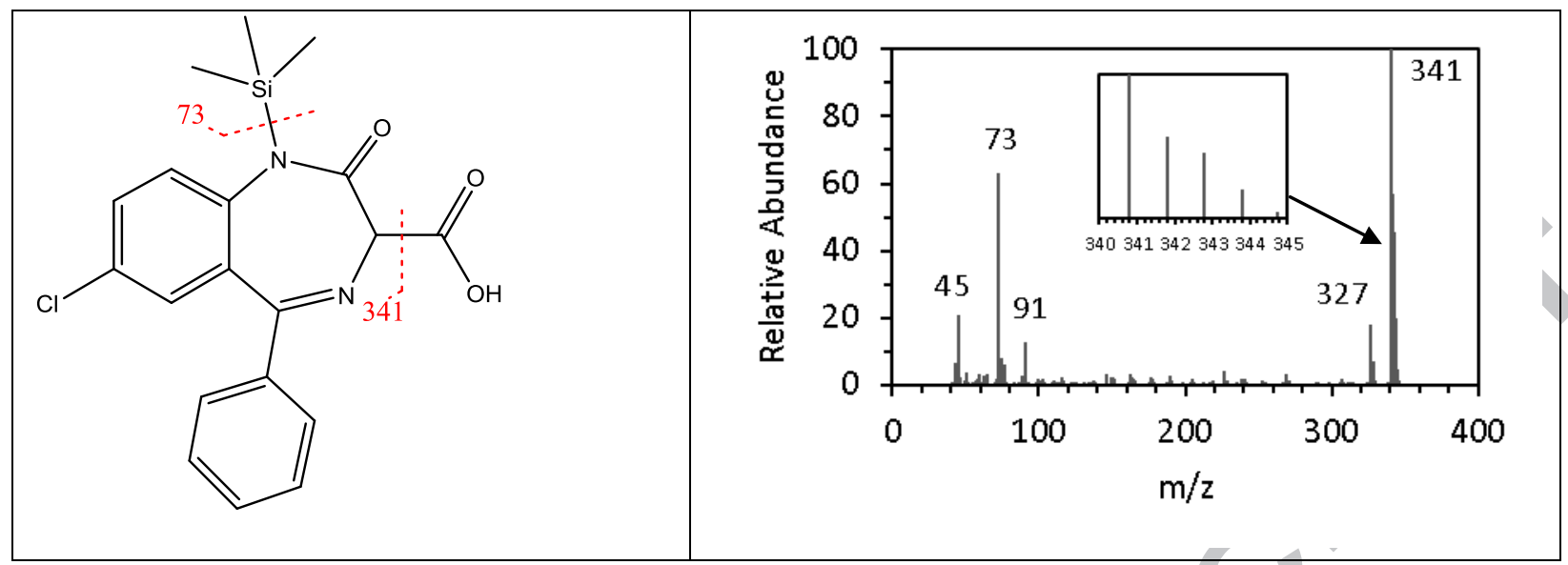

Figure 8. Structure and mass spectrum for lorazepam di-TMS (MW = 464) and chlorazepate TMS (MW 386).

\subsection{Zwitterions: Pregabalin, Vigabatrin, and Gabapentin}

The results for pregabalin were generally negative. The parent compound was not detected in the underivatized form. Derivatization using TFAA produced several chromatographic peaks, none of which were the expected derivative. The derivatization of pregabalin with BSTFA produced several peaks, including mono-, di-, and tri-TMS derivatives of pregabalin. Due to peaks for multiple products from one drug and a peak for underivatized pregabalin, the derivatization with BSTFA was considered unsuccessful. Derivatization with DMF-DMA also proved unsuccessful, with the target derivative detected but accompanied by multiple unidentified peaks.

Vigabatrin was not detectable in the underivatized form and derivatization of vigabatrin with TFAA produced no results. When silylated with BSTFA, a single chromatographic peak was produced (Figure 9). This derivative was not in the libraries, but its structure was elucidated by the fragmentation pattern (see Figure 10). Loss of a methyl group produced a (M-15) ion at $m / z 186$. From the $m / z 186$ fragment, a loss of 17 was observed in the form of ammonia following hydrogen rearrangement [26]. The peak at $\mathrm{m} / z 75$ is $\mathrm{C}_{2} \mathrm{H}_{7} \mathrm{SiO}$ [26]. The base peak of $m / z 56$ was formed by alpha cleavage at the nitrogen.

Derivatization with DMF-DMA produced a single chromatographic peak (Figure 9). Vigabatrin underwent the same derivatization process with DMF-DMA as gabapentin. This derivative was not in the libraries, but its structure was elucidated by the fragmentation pattern (see Figure 10). The molecular ion of $m / z$ 198 was seen. The ion at $m / z 167$ was a result of the loss of the methoxy group, the base peak of $m / z$ 111 was formed via alpha cleavage at the original nitrogen, and the $m / z 154$ ion was produced by alpha cleavage in the other direction losing the dimethylamine. 

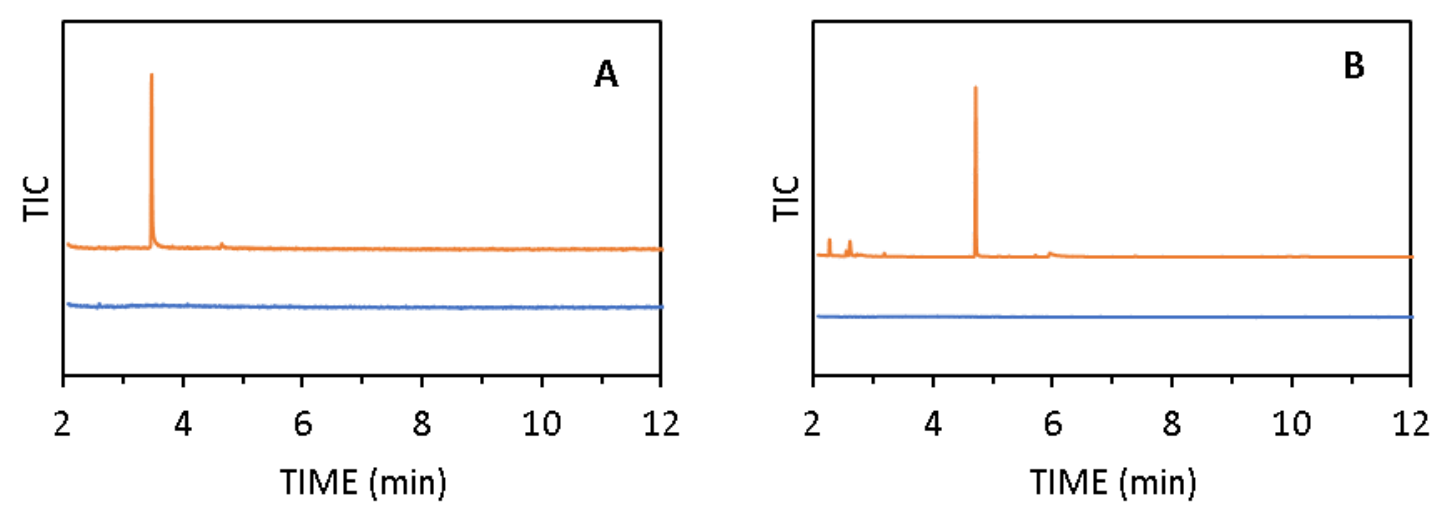

Figure 9. A) TIC of vigabatrin TMS (top) and vigabatrin underivatized (bottom), B) TIC of vigabatrin DMAM (top) and vigabatrin underivatized (bottom).

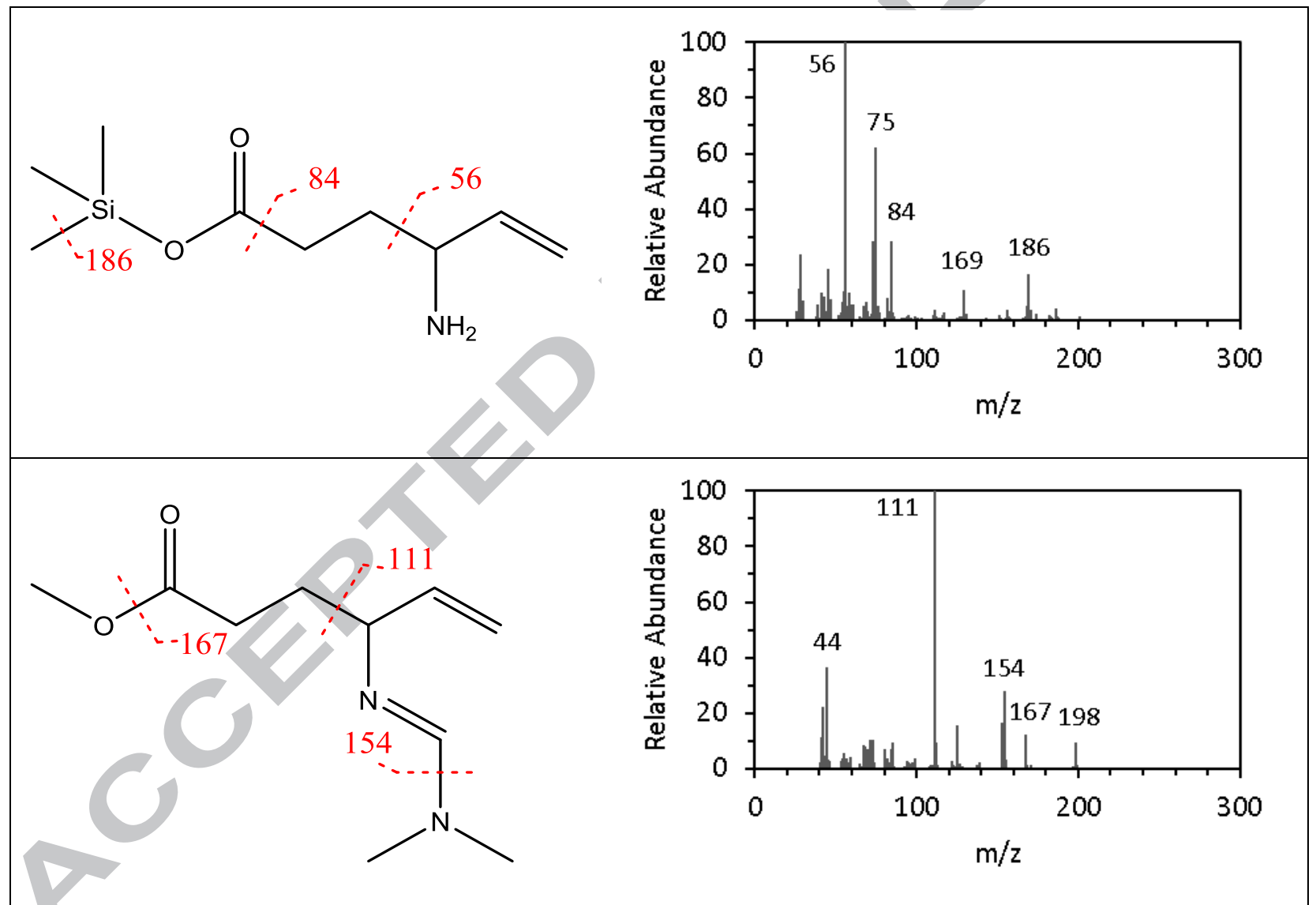

Figure 10. Structure and mass spectrum for vigabatrin TMS $(M W=201)$ and vigabatrin DMAM $(M W=$ 198).

Although potentially problematic for analysis by GC-MS, underivatized gabapentin produced a single chromatographic peak (Figure 11) with a mass spectrum that was identified with a mass spectral library search. Derivatization with TFAA produced a single chromatographic peak. This derivative was not in the libraries, but its mass spectrum was identified by analyzing the fragmentation pattern (see Figure 12). One of the hydrogens on the amine nitrogen was replaced by a trifluoroacetyl group. The molecular ion 
was not seen on the mass spectrum. Instead, an M-18 $(\mathrm{m} / \mathrm{z} 249)$ peak was seen arising from the loss of water. The $m / z 180$ base peak was formed by the further loss of $\mathrm{CF}_{3}$. Derivatization of gabapentin with BSTFA was not successful. No target derivative was formed. Derivatization of gabapentin by DMF-DMA produced a single chromatographic peak (Figure 11). This derivative was not in the libraries, but its mass spectrum was interpreted by analyzing the fragmentation pattern (see Figure 12). Gabapentin contains both a primary amine and a carboxylic acid, so its derivatization was directly analogous to that of primary amino acids [12]. The amine underwent the same process as that in amphetamine and $2 \mathrm{C}-1$, and the carboxylic acid was methylated in one step, resulting in an $\mathrm{N}, \mathrm{N}$-dimethylaminomethylene methyl ester [12]. The molecular ion $(\mathrm{m} / \mathrm{z} 240)$ was seen in very small abundance in the mass spectrum. A fragment was seen at $m / z 209$ which was the result of the molecular ion losing 31 mass units in the form of a methoxy radical. The base peak of $\mathrm{m} / z 85$ resulted from alpha cleavage at the original nitrogen. Loss of the dimethylamino group resulted in an $\mathrm{m} / \mathrm{z} 44$ and an $\mathrm{m} / \mathrm{z} 196$ fragment.
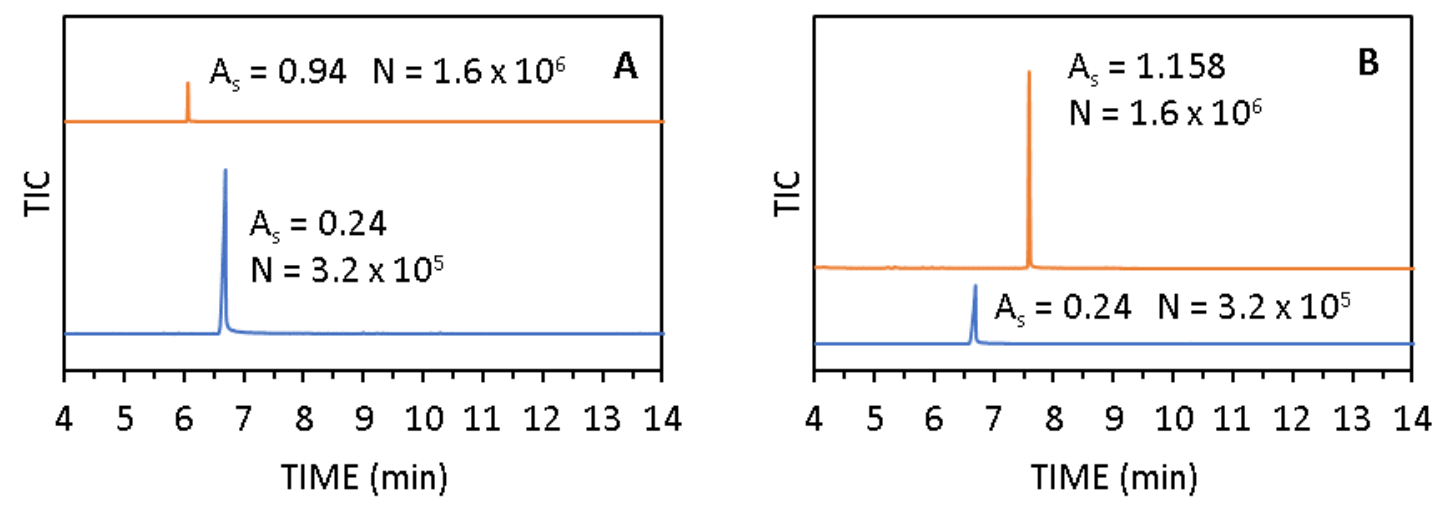

Figure 11. A) TIC of gabapentin TFA (top) and gabapentin underivatized (bottom), B) TIC of gabapentin DMAM (top) and gabapentin underivatized (bottom). $A_{s}=$ asymmetry factor. $\mathrm{N}=$ number of theoretical plates.

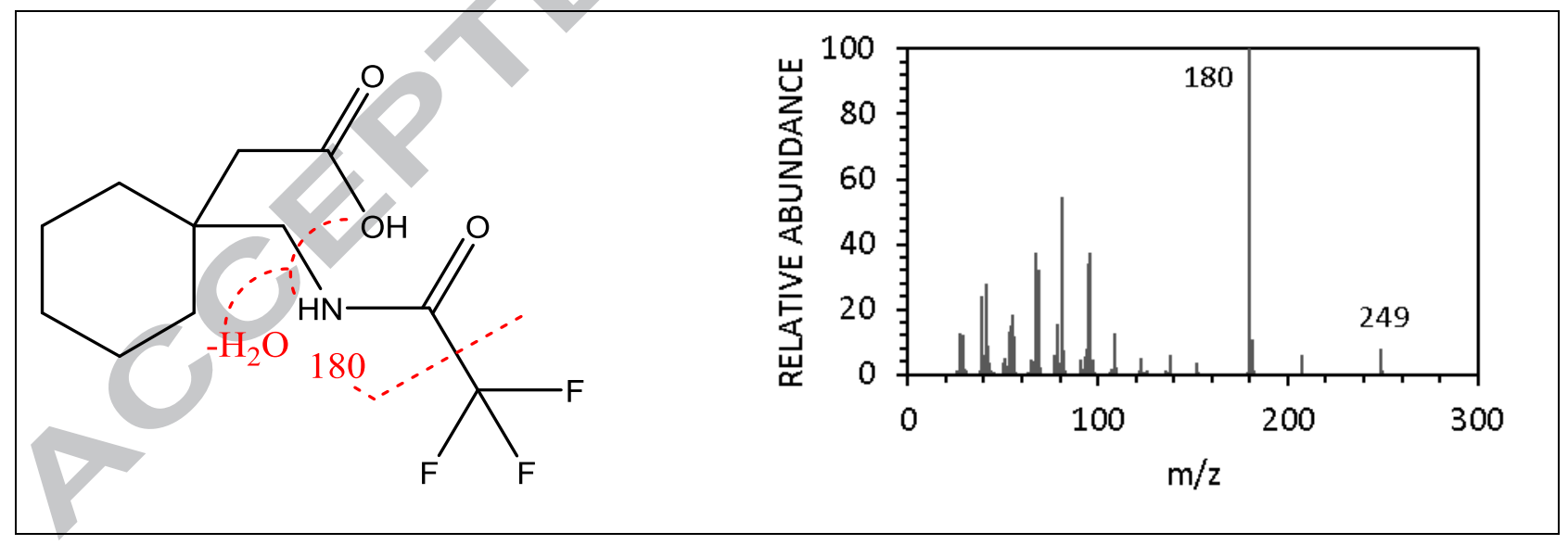



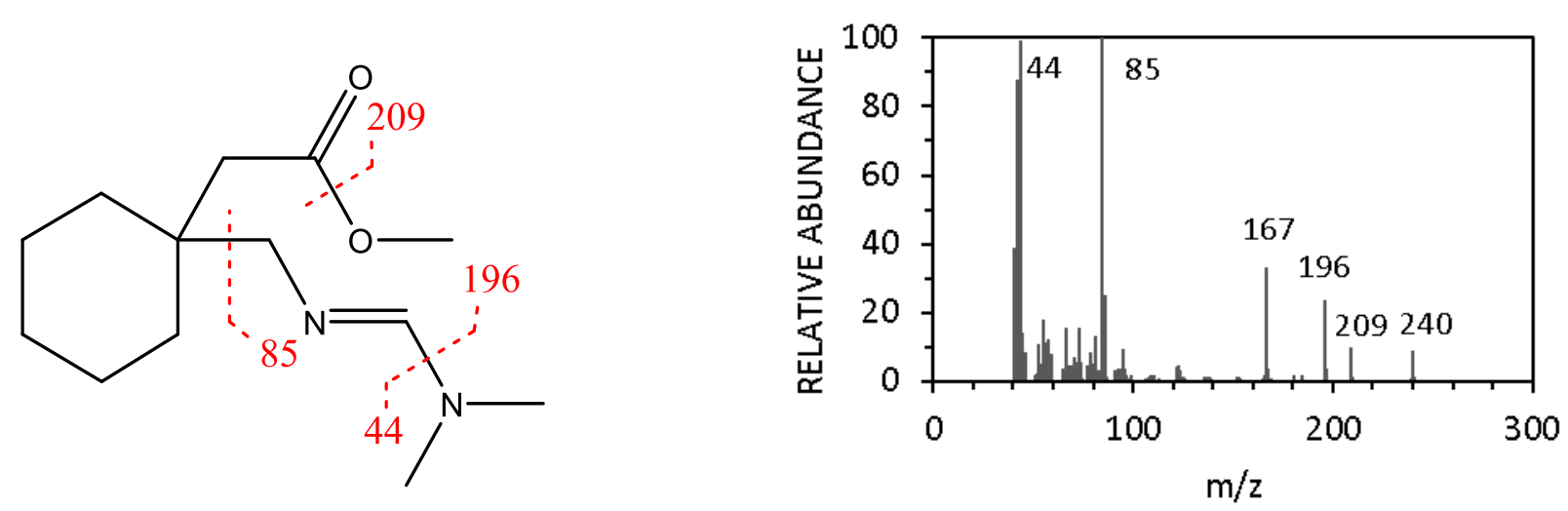

Figure 12. Structure and mass spectrum for gabapentin TFA (MW $=267)$ and gabapentin DMAM $(M W=$ 240).

\subsection{Effect of Derivatization on Chromatography}

The effects of derivatization on amphetamine, $2 \mathrm{C}-\mathrm{I}$ and gabapentin are displayed below as radar charts [27], where each peak is scored along three axes: area, theoretical plates $(N)$ and symmetry, which was calculated from the absolute value of $\left(A_{s}-1\right)$. Each variable was normalized to one based upon the highest value of $\mathrm{N}$, area, and symmetry for the drug and its derivatives. Therefore, the area inscribed by a particular drug/derivative is directly related to better chromatographic performance.

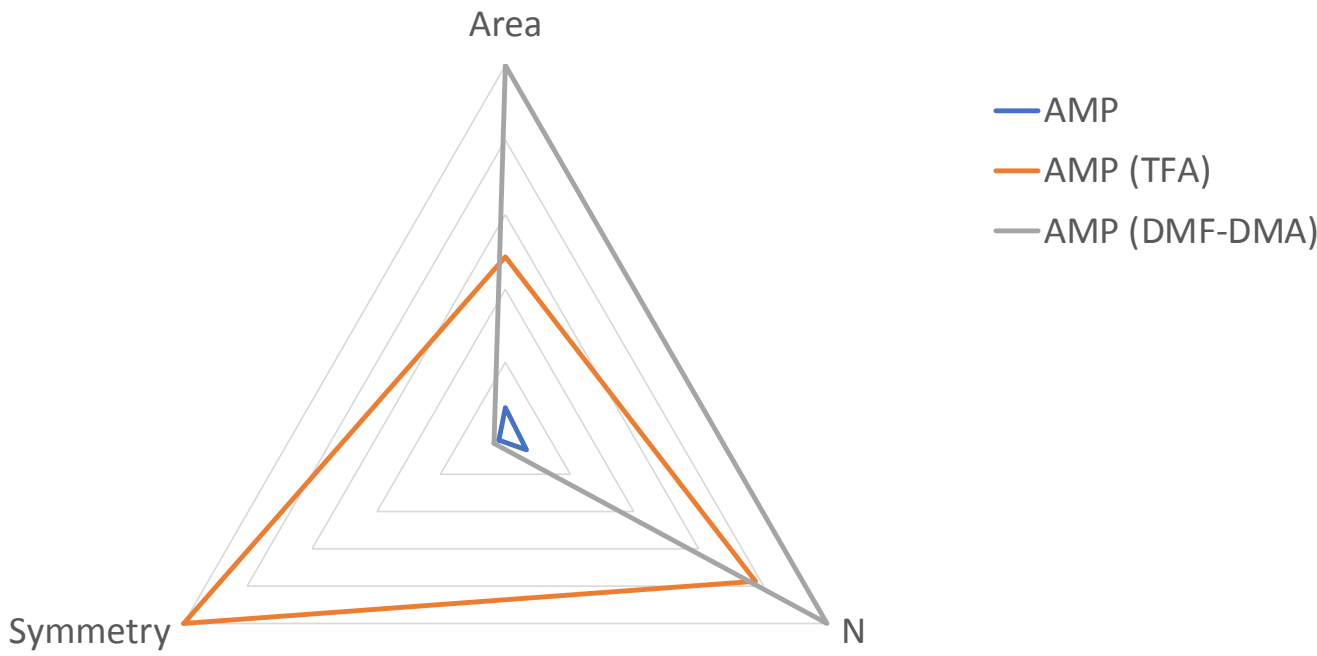

Figure 13. Effect of derivatization on the symmetry, area and theoretical plates for amphetamine. 


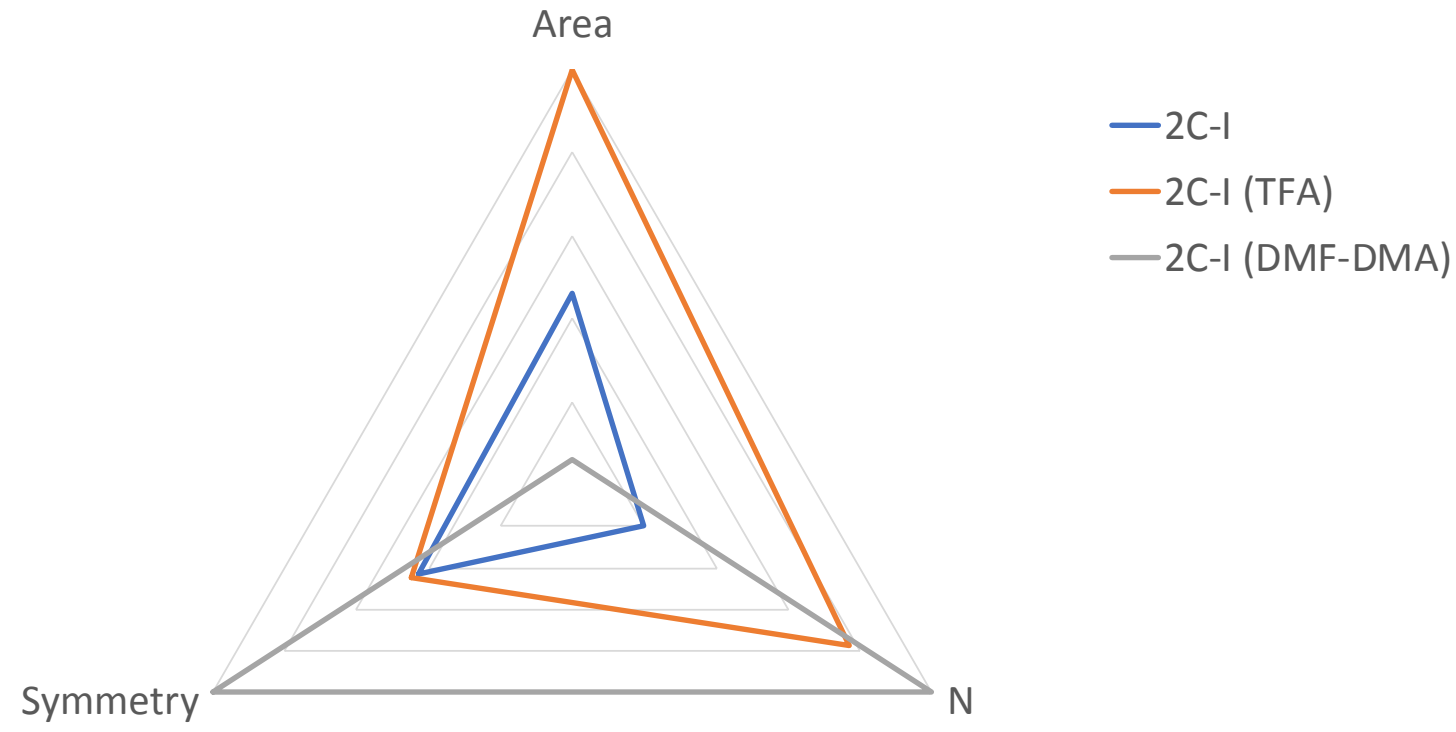

Figure 14. Effect of derivatization on the symmetry, area and theoretical plates for 2C-I.

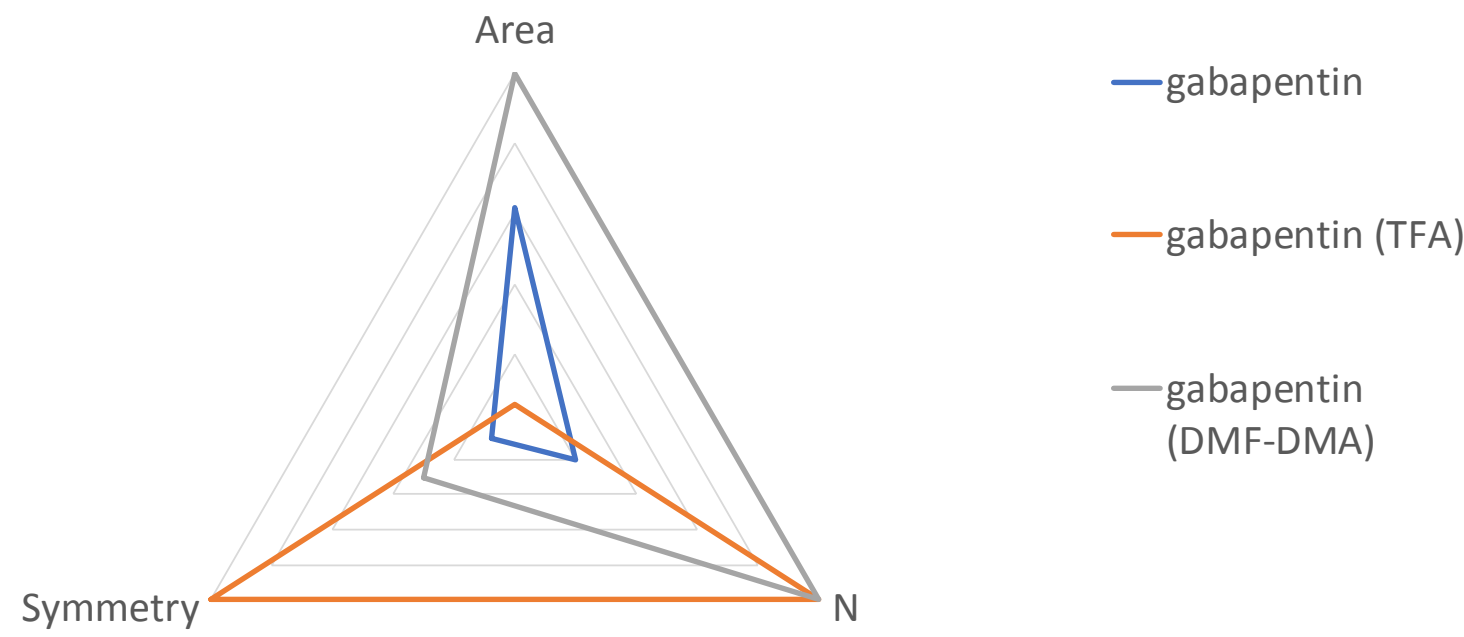

Figure 15. Effect of derivatization on the symmetry, area and theoretical plates for gabapentin.

When the data is grouped as drugs "as is" $(n=3)$, TFA derivatives $(n=3)$ and DMF-DMA derivatives $(n=$ $3)$, multi-dimensional testing is possible. A correlation matrix showed that there was no significant correlation between $\mathrm{N} /$ area and $\mathrm{N} /$ symmetry. There was a modest negative correlation $(r=-0.36)$ between area and symmetry. This is consistent with "peak overloading" at high concentrations. In this case, peaks become asymmetric due to the solute deviating from a linear isotherm that exists between the concentration of the solute in the stationary phase and the concentration of the solute in the mobile phase.

Multi-variate statistical tests (e.g., Box test and Kulback's test) concluded that the within-class covariance matrices were not equal $(p<0.0001)$. Testing of the means vectors of the three classes 
(Wilke's lambda test) yielded a p value of 0.096 , hence the confidence level was only $90.4 \%$. Given that each class only had three members, additional replicates would be needed to yield a higher degree of confidence.

\section{Conclusions}

Basic and zwitterionic drugs can be amongst the most difficult to analyze via GC-MS due to their thermal instability and non-ideal behavior resulting in broad, asymmetric peaks. These issues can be overcome with derivatization. A summary of the drugs and derivatizations discussed in this paper appears in Table 2.

[INSERT TABLE 2 HERE]

Several drugs (e.g., amphetamine, 2C-I, gabapentin, and lorazepam) were successfully analyzed by GCMS without modification. However, the number of theoretical plates achieved was dramatically increased by derivatization and peak symmetry was greatly improved. Analysis of vigabatrin, pregabalin and clorazepate yielded negative results, as no peaks were formed.

Among the drugs of interest, amphetamine and 2C-I were readily derivatized with TFAA. The derivatives produced more intense and narrower chromatographic peaks than their underivatized forms. Gabapentin was also successfully derivatized with TFAA, but the resulting chromatographic peak was smaller in magnitude than that of the underivatized drug. Lorazepam, vigabatrin, pregabalin, and clorazepate were not successfully derivatized by TFAA - lorazepam and vigabatrin produced no chromatographic peaks, while pregabalin and clorazepate both produced multiple peaks, none of with were the target derivatives.

Derivatization with BSTFA + 1\% TMCS produced single chromatographic peaks for lorazepam, vigabatrin, and clorazepate. The derivatization was incomplete for amphetamine and wholly unsuccessful for gabapentin, producing no derivative. BSTFA derivatization yielded multiple derivatives with one, two, or even three TMS groups for $2 \mathrm{C}-\mathrm{I}$ and pregabalin.

As previously discussed, methyl-N-t-butyldimethylsilyltrifluoroacetamide (MTBSTFA) and other silylation reagents that replace active hydrogens with larger t-butyldimethylsilyl rather than trimethylsilyl groups generally take longer to form, but are more stable. Additionally, the t-butyldimethylsilyl group is more sterically hindering than the trimethylsilyl group, so t-butyldimethylsilyl reagents will likely form only one derivative with primary amines. It is therefore possible that reactions with BSTFA that were unsuitable due to multiple products or for which no derivative was detected here may produce useful results when reacted with larger silylation reagents such as MTBSTFA.

Lastly, DMF-DMA proved an effective new method for the derivatization of amphetamine, 2C-I, gabapentin, and vigabatrin. The primary amine hydrogens in amphetamine and $2 \mathrm{C}-\mathrm{I}$ were replaced with a DMAM group. Gabapentin and vigabatrin, containing both a primary amine and a carboxyl group, underwent the addition of a methyl group and a DMAM group.

\section{Acknowledgements}

The authors would like to acknowledge Donna Roskowski of the FIS Program at IUPUI for suggesting the drugs discussed in this paper as well as for helpful suggestions on the GC-MS method. The authors acknowledge the National Institute of Justice for providing funding for this project (Award No. 2015-DN- 
BX-K058). The opinions, findings, and conclusions expressed in this publication are those of the authors and do not necessarily reflect those of the Department of Justice. 


\section{References}

[1] F. Smith, J.A. Siegel, Handbook of Forensic Drug Analysis, 1 ed., Elsevier Science, Burlington, MA, 2004.

[2] D.-L. Lin, S.-M. Wang, C.-H. Wu, B.-G. CHen, R.H. Liu, Chemical Derivatization for the Analysis of Drugs by GC-MS - A Conceptual Review, Journal of Food and Drug Analysis 16(1) (2008) 1-10.

[3] C. Jurado, M. Gimenez, T. Soriano, M. Menendez, M. Repetto, Rapid analysis of amphetamine, methamphetamine, MDA, and MDMA in urine using solid-phase microextraction, direct on-fiber derivatization, and analysis by GC-MS, Journal of analytical toxicology 24(1) (2000) 11-16.

[4] A. Hulshoff, H. Lingeman, Derivatization reactions in the gas-liquid chromatographic analysis of drugs in biological fluids, Journal of pharmaceutical and biomedical analysis 2(3-4) (1984) 337-380.

[5] T.A. Brettell, Analysis of N-mono-trifluoroacetyl derivatives of amphetamine analogues by gas chromatography and mass spectrometry, Journal of Chromatography A 257 (1983) 45-52. [6] M.K.R. Mudiam, A. Chauhan, R. Jain, R. Ch, G. Fatima, E. Malhotra, R. Murthy, Development, validation and comparison of two microextraction techniques for the rapid and sensitive determination of pregabalin in urine and pharmaceutical formulations after ethyl chloroformate derivatization followed by gas chromatography-mass spectrometric analysis, Journal of pharmaceutical and biomedical analysis 70 (2012) 310-319.

[7] K. Sowjanya, J. Thejaswini, B. Gurupadayya, P. Raja, Quantitative Determination of Pregabalin by Gas Chromatography using Ethyl Chloroformate as a Derivatizing Reagent in Pure and Pharmaceutical Preparation, Ind. Drugs 48(3) (2011) 43-47.

[8] T. Hlozek, M. Bursova, P. Coufal, R. Cabala, Gabapentin, pregabalin and vigabatrin quantification in human serum by GC-MS after hexyl chloroformate derivatization, J. Anal. Toxicol. 40(9) (2016) 749-753.

[9] C. Schummer, O. Delhomme, B.M. Appenzeller, R. Wennig, M. Millet, Comparison of MTBSTFA and BSTFA in derivatization reactions of polar compounds prior to GC/MS analysis, Talanta 77(4) (2009) 1473-1482.

[10] F. Orata, Derivatization reactions and reagents for gas chromatography analysis, Advanced Gas Chromatography-Progress in Agricultural, Biomedical and Industrial Applications, InTech2012.

[11] J.-P. Thenot, E. Horning, M. Stafford, M. Horning, Fatty acid esterification with N, Ndimethylformamide dialkyl acetals for GC analysis, Analytical Letters 5(4) (1972) 217-223. [12] J. Thenot, E. Horning, Amino acid N-dimethylaminomethylene alkyl esters. New derivatives for GC and GC-MS studies, Analytical Letters 5(8) (1972) 519-529.

[13] M.F. Grubb, P.S. Callery, Derivatization of N-methyl and cyclic amino acids with dimethylformamide dimethyl acetal, Journal of Chromatography A 469 (1989) 191-196.

[14] H. Kataoka, K. Kijima, Analysis of heterocyclic amines as their N-dimethylaminomethylene derivatives by gas chromatography with nitrogen-phosphorus selective detection, Journal of Chromatography A 767(1-2) (1997) 187-194.

[15] N. Kostic, Y. Dotsikas, A. Malenovic, Critical review on the analytical methods for the determination of zwitterionic antiepileptic drugs-vigabatrin, pregabalin, and gabapentin-in bulk and formulations, Instrum. Sci. Technol. 42(4) (2014) 486-512. 
[16] D.S. Theobald, M. Pütz, E. Schneider, H.H. Maurer, New designer drug 4- iodo- 2, 5- dimethoxy- $\beta$ - phenethylamine $(2 \mathrm{C}$ - I): studies on its metabolism and toxicological detection in rat urine using gas chromatographic/mass spectrometric and capillary electrophoretic/mass spectrometric techniques, Journal of mass spectrometry 41(7) (2006) 872-886.

[17] F. Van Lente, V. Gatautis, Cost-efficient use of gas chromatography-mass spectrometry: a "piggyback" method for analysis of gabapentin, Clinical chemistry 44(9) (1998) 2044-2045.

[18] F. Centini, A. Masti, I.B. Comparini, Quantitative and qualitative analysis of MDMA, MDEA, MA and amphetamine in urine by head-space/solid phase micro-extraction (SPME) and GC/MS, Forensic science international 83(3) (1996) 161-166.

[19] D. Blachut, M. Bykas-Strekowska, E. Taracha, B. Szukalski, Application of gas chromatography/mass spectrometry (GC/MS) to the analysis of benzodiazepines, Z Zagadnien Nauk Sadowych 59 (2004) 5-37.

[20] Y. Nakahara, K. Takahashi, M. Shimamine, Y. Takeda, Hair analysis for drug abuse: I. Determination of methamphetamine and amphetamine in hair by stable isotope dilution gas chromatography/mass spectrometry method, Journal of Forensic Science 36(1) (1991) 70-78. [21] H.R. Lin, A.C. Lua, Simultaneous determination of amphetamines and ketamines in urine by gas chromatography/mass spectrometry, Rapid communications in mass spectrometry 20(11) (2006) 1724-1730.

[22] M. Kushnir, J. Crossett, P. Brown, F. Urry, Analysis of gabapentin in serum and plasma by solid-phase extraction and gas chromatography-mass spectrometry for therapeutic drug monitoring, Journal of analytical toxicology 23(1) (1999) 1-6.

[23] P.E. Roman, J.A. Knapp, C.K. Horn, S.L. Stillman, J.E. Evans, D.P. Arfsten, Comparison of LC-MS-MS and GC-MS analysis of benzodiazepine compounds included in the drug demand reduction urinalysis program, J. Anal. Toxicol. 40(3) (2016) 201-207.

[24] A.V. de Bairros, R.M. de Almeida, L. Pantaleão, T. Barcellos, S.M.e. Silva, M. Yonamine, Determination of low levels of benzodiazepines and their metabolites in urine by hollow-fiber liquid-phase microextraction (LPME) and gas chromatography-mass spectrometry (GC-MS), Journal of Chromatography B 975(Supplement C) (2015) 24-33.

[25] R.H. Liu, D.V. Canfield, S.-M. Wang, Quantitation and mass spectrometric data of drugs and isotopically labeled analogs, CRC Press2009.

[26] F.W. McLafferty, Interpretation of Mass Spectra, Fourth ed., University Science Books, Sausalito, California, 1993.

[27] M.J. Saary, Radar plots: a useful way for presenting multivariate health care data, Journal of clinical epidemiology 61(4) (2008) 311-317. 
Table 1. References in which the zwitterions and primary amines presented in this work were successfully detected by GC-MS "as is" or using a derivatization agent of the category indicated.

\begin{tabular}{|c|c|c|c|c|}
\hline Drug & Underivatized & Acylation & Silylation & Alkylation \\
\hline Amphetamine & [17] & $\begin{array}{l}\text { TFAA }[\underline{2}, \underline{19}, \underline{20}] \\
\text { MBTFA }[\underline{4}]\end{array}$ & & $\begin{array}{l}\text { Cyclohexanone [24] } \\
\text { Trifluoroacetylacetone } \\
{[\underline{25}]}\end{array}$ \\
\hline $2 \mathrm{C}-\mathrm{I}$ & {$[\underline{15}]$} & TFAA [15] & & $>>$ \\
\hline Lorazepam & [18] & TFAA [르] & $\begin{array}{l}\text { MTBSTFA } \\
{[\underline{22}][\underline{23}]}\end{array}$ & \\
\hline Clorazepate & & BSTFA + TMCS [26] & & lodomethane [26] \\
\hline Gabapentin & {$[\underline{16}, \underline{18}]$} & TFAA $[\underline{7}, \underline{27}]$ & MTBSTFA [21] & $\mathrm{MeOH} / \mathrm{HCl}[\underline{27}]$ \\
\hline Vigabatrin & & $\begin{array}{l}\text { Hexyl chloroformate } \\
\text { [7] }\end{array}$ & & \\
\hline Pregabalin & & $\begin{array}{l}\text { Ethyl chloroformate [ } \underline{5} \text {, } \\
\underline{6}] \\
\text { Hexyl chloroformate } \\
{[\underline{7]}}\end{array}$ & & \\
\hline
\end{tabular}

Trifluoroacetic anhydride (TFAA)

N-Methyl-bis(trifluoroacetamide) (MBTFA)

$\mathrm{N}$-tert-Butyldimethylsilyl-N-methyltrifluoroacetamide (MTBSTFA) 
Table 2. A summary of the results of this work. " + " (green) indicates the formation of a single chromatographic peak that could be unambiguously identified, " 0 " (yellow) indicates that multiple peaks were formed and identified as separate derivatives of the same parent compound, and "_" (orange) indicates that no relevant peak was formed.

\begin{tabular}{|c|c|c|c|c|}
\hline Drug & Underivatized & TFAA & BSTFA & DMF-DMA \\
\hline Amphetamine & + & + & 0 & + \\
\hline $2 C-I$ & + & + & 0 & + \\
\hline Lorazepam & + & - & + & - \\
\hline Clorazepate & - & - & + & - \\
\hline Gabapentin & + & + & - & + \\
\hline Vigabatrin & 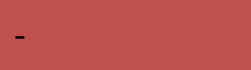 & - & + & + \\
\hline Pregabalin & - & - & 0 & 0 \\
\hline
\end{tabular}




\section{Graphical abstract}
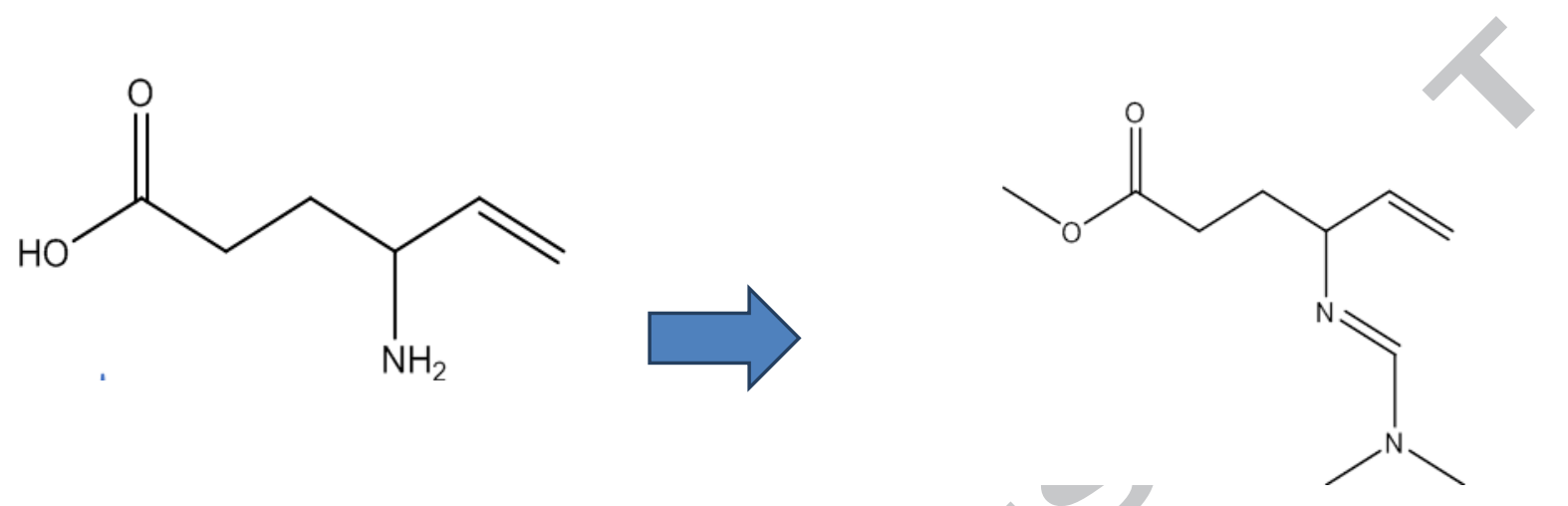


\section{Highlights}

- Many drugs are not amenable to GC-MS due to thermal instability, non-ideal interactions in the column, or both.

- Three different derivatization agents were tested for effectiveness with primary amines (i.e., amphetamine and $2 \mathrm{C}-\mathrm{I}$ ) and zwitterions (i.e., gabapentin, lorazepam, vigabatrin, pregabalin, and clorazepate).

- Dimethylformamide-dimethyl acetal (DMF-DMA) formed dimethylaminomethylene derivatives with several primary amines and zwitterions. 